\title{
Interference Alignment for Cooperative MIMO Femtocell Networks
}

\author{
Basak Guler and Aylin Yener \\ Electrical Engineering Department \\ The Pennsylvania State University, University Park, PA 16801 \\ bxg215@psu.edu_yener@ee.psu.edu
}

\begin{abstract}
This paper proposes a method for applying the idea of Interference Alignment (IA) in femtocell networks. In order to manage the uplink interference caused by macrocell users at the femtocell base stations (FBS), cooperation between macrocell users with the closest femtocell base stations could be used to align the received signals of macrocell users in the same subspace at multiple FBS simultaneously. We develop a method to apply IA while providing the QoS requirements of macrocell users, in terms of minimum received SINR at the macrocell base station (MBS). With this approach, the BER performance of femtocell users is shown to improve, while maintaining the quality of the communication channel of macrocell users.
\end{abstract}

\section{INTRODUCTION}

Next generation wireless networks are designed to provide high data rates to meet subscriber demands. Femtocells are a promising direction to increase the data rate for home users while reducing the load on the cellular (macrocell) network [1]. They require no infrastructure as they are plug and play devices that are connected to the conventional internet backhaul [2]. Femtocells operate in the licenced band, and consequently have to share the radio resources and coexist with the cellular network. Solutions proposed to guarantee coexistence range from partitioning the frequency resources between the two networks, to allowing cellular (macrocell) users to be served by femtocell base stations [1]. Management of cross interference in this two-tier network is of utmost importance. In the uplink, in particular, a macrocell user operating in the same band as femtocell users may cause unacceptably high interference levels, if it is close to the femtocell base station supporting the aforementioned femtocell users, and far away from its own macrocell base station. Additionally, the fact that femtocells can be deployed in an ad hoc fashion anywhere within a macrocell (and can be removed as easily) adds to the challenge of interference management and renders jointly optimal design of the two networks impractical.

Interference management has been an important design element for multiuser systems in the past two decades. Judicious receiver design for CDMA systems provides effective interference cancellation [3]. Besides multiuser detection, power control [4], and joint design of transmitters and receivers [5], [6], [16] offer optimal interference mitigation in interference limited systems. While the aforementioned techniques have been primarily designed for multi-transmitter single receiver (multiple access) systems, interference alignment has recently been proposed for multi-transmitter multi-receiver (interference) networks and has been shown to achieve the maximum degrees of freedom for the $K$-user interference channel [7]. For practical scenarios, distributed algorithms have been proposed for interference alignment; these include minimizing the leakage interference and using channel reciprocity [8], minimizing MSE [9], or alternating minimization [10].

In this paper, we take the viewpoint of managing the interference caused by the macrocell users to the uplinks of femtocells in their vicinity by aligning their signals. Consequently, the quality of service/performance of the femtocell users is improved without diminishing the quality of service of the macrocell users. We leverage the recent advances in interference alignment and base station cooperation (for the femtocells) in order to put forward a practically relevant yet close to optimal design of this two-tier network.

In order to manage the uplink interference caused by the macrocell users at the femtocell base stations (FBS), joint detection or interference cancellation may be used. Joint detection may not be preferred due to privacy issues and the limited backhaul provided by the Internet service provider (ISP) to the femtocells. Interference cancellation methods such as zero forcing requires as many antennas at the FBS as the number of signals to be cancelled, which may be impractical in dense urban areas since only a limited number of antennas can be employed at the FBSs. We posit that a more viable alternative is by means of coordination between multiple FBS and the macrocell users that are causing high interference to this group of FBSs. Specifically, using the principle of interference alignment (IA), we can align the received signals from macrocell users in a lower dimensional subspace at multiple FBSs simultaneously, and use the remaining degree of freedoms to improve the performance of the femtocell users. While interference alignment helps the femtocell users to eliminate macrocell interference, this should not be at the expense quality of service (QoS) for the macrocell users. Our approach is that macrocell users apply interference alignment with individual SINR constraints at the MBS, thus making sure their QoS requirements are met. To solve this problem, we propose an algorithm that uses successive semi-definite programming (SDP) relaxations, which will be referred as SDP-IA algorithm. After interference alignment, a precodingdecoding scheme is used at the FBSs which minimizes the sum MSE of the femtocell users with coordinated zero forc- 


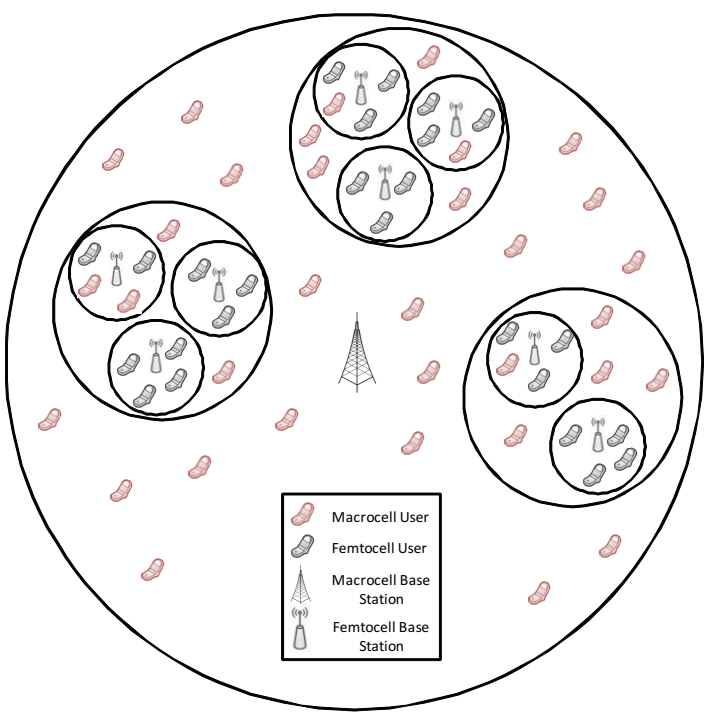

Fig. 1. System Model with a single MBS and 3 FBS groups

ing to eliminate macrocell interference.The numerical results demonstrate that the benefits of the proposed IA algorithm, and that these benefits increase as the number of interfering macrocell users increase. The number of macrocell users that can be aligned simultaneously depends on the minimum SINR requirements at the MBS, more users can be aligned when the minimum SINR requirements are decreased.

The remainder of the paper is organized as follows: In Section II, we introduce the system model. Interference alignment for macrocell users is presented in Section III. Section IV describes the precoding and decoding scheme for femtocell users. In Section V, the numerical results and simulations are discussed. We conclude the paper in Section VI. The notation used in the paper is as follows: We use lower (upper) bold case letters for vectors (matrices). $\mathbf{X}^{H}$ is used to denote the Hermitian transpose, $\mathbf{X}^{\dagger}$ as the pseudo-inverse of matrix $\mathbf{X}$, and $\otimes$ for the Kronecker product. Finally, trace $(\mathbf{X})$ represents the trace of matrix $\mathbf{X}$.

\section{SySTEM MOdeL}

We consider an uplink femtocell network as shown in Fig.1 consisting of a macrocell base station (MBS) at the center with $N_{o}$ receive antennas. The macrocell coverage area is partitioned into smaller cells of fixed radius in which the mobile users and base stations can cooperate with each other. Suppose such a group consists of $\mathrm{F}$ femtocell base stations (FBS), with $U_{f}$ users in the $f^{\text {th }}$ femtocell (FU) and $\mathrm{M}$ macrocell users (MU). We have $N_{t}$ transmit antennas at each mobile device and $N_{f}$ receive antennas at the $f^{t h}$ FBS. Then the signal received at the $k^{\text {th }}$ FBS is given by

$$
\begin{aligned}
\mathbf{y}_{k}= & \sum_{i=1}^{U_{k}} \mathbf{H}_{k i}^{k} \mathbf{w}_{i}^{k} s_{i}^{k} \\
& +\sum_{\substack{f=1 \\
f \neq k}}^{F} \sum_{u=1}^{U_{f}} \mathbf{H}_{k u}^{f} \mathbf{w}_{u}^{f} s_{u}^{f}+\sum_{m=1}^{M} \mathbf{H}_{k m}^{o} \mathbf{w}_{m}^{o} s_{m}^{o}+\mathbf{n}_{k}
\end{aligned}
$$

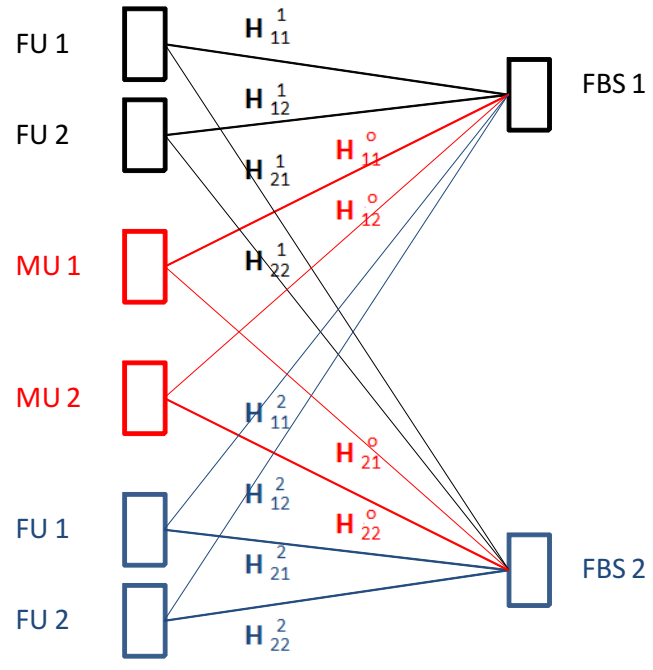

Fig. 2. Model for a case of 2 macrocell users and 2 FBSs, each with 2 users

where $\mathbf{H}_{k u}^{f}$ denotes the channel from the $u^{t h}$ user of the $f^{t h}$ femtocell to the $k^{t h}$ FBS, $\mathbf{H}_{k m}^{o}$ is the channel from the $m^{t h}$ MU to the $k^{t h}$ FBS, $\mathbf{w}_{u}^{f}$ and $s_{u}^{f}$ are the precoding vector and the message bit of the $u^{t h}$ user of the $f^{t h}$ femtocell, $\mathbf{w}_{m}^{o}$ and $s_{m}^{o}$ are the precoding vector and message bit of the $m^{t h} \mathrm{MU}, \mathbf{n}_{k}$ is a vector of independent zero mean Gaussian random variables with $E\left\{\mathbf{n}_{k} \mathbf{n}_{k}{ }^{H}\right\}=\sigma^{2} \mathbf{I}$. The channels considered are Rayleigh fading channels and the path loss is modeled using the ITU-R channel model [11]. We used rank 1 precoders to reduce the complexity of the algorithm and to avoid feasibility problems due to the large number of users. We assume $s_{u}^{f}$ and $s_{m}^{o}= \pm 1$ for $u=1, \ldots, U_{f}, f=1, \ldots, F$, and $m=1, \ldots, M$. An example model is given in Fig.2 for 2 macrocell users and 2 FBSs, each with 2 users.

\section{Interference Alignment with Successive SDP RELAXATIONS}

For simplicity, we will neglect the uplink interference caused at a FBS by the users of other femtocells, and consider only the (dominant) interference caused by the macrocell users. We will use the condition for interference alignment proposed in [12]:

$$
\begin{aligned}
& \mathbf{H}_{11}^{o} \mathbf{w}_{1}^{o}=\alpha_{12} \mathbf{H}_{12}^{o} \mathbf{w}_{2}^{o}=\cdots=\alpha_{1 M} \mathbf{H}_{1 M}^{o} \mathbf{w}_{M}^{o} \\
& \mathbf{H}_{21}^{o} \mathbf{w}_{1}^{o}=\alpha_{22} \mathbf{H}_{22}^{o} \mathbf{w}_{2}^{o}=\cdots=\alpha_{2 M} \mathbf{H}_{2 M}^{o} \mathbf{w}_{M}^{o} \\
& \quad \vdots \\
& \mathbf{H}_{F 1}^{o} \mathbf{w}_{1}^{o}=\alpha_{F 2} \mathbf{H}_{F 2}^{o} \mathbf{w}_{2}^{o}=\cdots=\alpha_{F M} \mathbf{H}_{F M}^{o} \mathbf{w}_{M}^{o}
\end{aligned}
$$

where $\alpha_{f m}$ is a constant and the equations denote that all interfering users span the same column space at each FBS. That is, each interfering signal is represented by a linear combination of other interfering signals, represented by different coefficients. Using the precoders and the associated coefficients, expressions (2)-(4) can be combined in a single matrix representation as in (5), as proposed in [12]. Then the condition of perfect interference alignment is equal to the expression being equal to zero (5). Therefore, one approach for 
finding the interference aligning precoding matrices is to make the norm of this expression as close to zero as possible as in (6), from which follows the notion of least squares approach for interference alignment, proposed in [12].

$$
\mathrm{Hw}=\mathbf{0}
$$

where

$$
\mathbf{H}=\left[\begin{array}{ccccc}
\mathbf{H}_{11}^{o} & -\alpha_{12} \mathbf{H}_{12}^{o} & 0 & \ldots & 0 \\
\mathbf{H}_{11}^{o} & 0 & -\alpha_{13} \mathbf{H}_{13}^{o} & \ldots & 0 \\
\vdots & \vdots & \vdots & \ddots & \vdots \\
\mathbf{H}_{11}^{o} & 0 & 0 & \ldots & -\alpha_{1 M} \mathbf{H}_{1 M}^{o} \\
\vdots & \vdots & \vdots & \ddots & \vdots \\
\mathbf{H}_{F 1}^{o} & -\alpha_{F 2} \mathbf{H}_{F 2}^{o} & 0 & \ldots & 0 \\
\mathbf{H}_{F 1}^{o} & 0 & -\alpha_{F 3} \mathbf{H}_{F 3}^{o} & \ldots & 0 \\
\vdots & \vdots & \vdots & \ddots & \vdots \\
\mathbf{H}_{F 1}^{o} & 0 & 0 & \ldots & -\alpha_{F M} \mathbf{H}_{F M}^{o}
\end{array}\right]
$$$$
\mathbf{w}=\left[\begin{array}{llllll}
\mathbf{w}_{1}^{o T} & \mathbf{w}_{2}^{o T} & \mathbf{w}_{3}^{o T} & \ldots & \mathbf{w}_{M-1}^{o T} & \mathbf{w}_{M}^{o T}
\end{array}\right]^{T}
$$

We will follow this definition for interference alignment, however, our solution follows a SDP relaxation method to solve the norm minimization problem that satisfies the individual minimum SINR requirements for each macrocell user, which incorporates successive SDP relaxations [21] and rank-one approximation. The interference alignment problem in (5) can be regarded as a least squares (LS) problem [12]. In fact, (5) denotes a set of linear equations and the LS approach is a conventional method to approximate the solution. In order to satisfy QoS requirements, we define an individual SINR constraint for each macrocell user. The problem is thus given by:

$$
\begin{array}{ll}
\underset{\mathbf{w}_{1}^{o}, \ldots, \mathbf{w}_{M}^{o}}{\operatorname{minimize}} & \|\mathbf{H w}\| \\
\text { subject to } & S I N R_{i} \geq \gamma_{i} \\
& \left(\mathbf{w}_{i}^{o}\right)^{H} \mathbf{w}_{i}^{o} \leq \mathrm{P}_{i}^{o} \quad i=1, \ldots, M
\end{array}
$$

where $\mathrm{P}_{i}^{o}$ denotes the maximum transmit power of the $i^{t h}$ macrocell user, $\gamma_{i}$ denotes the minimum SINR threshold of the $i^{t h}$ macrocell user, and $S I N R_{i}$ is given as in (8).

$$
\operatorname{SIN} R_{i}=\frac{\left(\mathbf{w}_{i}^{o}\right)^{H}\left(\mathbf{H}_{o i}^{o}\right)^{H} \mathbf{H}_{o i}^{o} \mathbf{w}_{i}^{o}}{\sum_{\substack{n=1 \\ n \neq i}}^{M}\left(\mathbf{w}_{n}^{o}\right)^{H}\left(\mathbf{H}_{o n}^{o}\right)^{H} \mathbf{H}_{o n}^{o} \mathbf{w}_{n}^{o}+\mathbf{B}+\sigma^{2}}
$$

where

$$
\mathbf{B}=\sum_{f=1}^{F} \sum_{u=1}^{U_{f}}\left(\mathbf{w}_{u}^{f}\right)^{H}\left(\mathbf{H}_{o u}^{f}\right)^{H} \mathbf{H}_{o u}^{f} \mathbf{w}_{u}^{f}
$$

where $\mathbf{H}_{o n}^{o}$ denotes the channel from the $n^{t h}$ macrocell user to the MBS. Then the equivalent problem can be written as:

$$
\begin{array}{ll}
\underset{\mathbf{w}_{1}^{o}, \ldots, \mathbf{w}_{M}^{o}}{\operatorname{minimize}} & \operatorname{trace}(\mathbf{R W}) \\
\text { subject to } & \operatorname{trace}\left(\left(\widehat{\mathbf{R}}_{o i}-\gamma_{i} \sum_{n \neq i} \widehat{\mathbf{R}}_{o n}\right) \mathbf{W}\right) \geq \gamma_{i} \sigma^{2}
\end{array}
$$

$$
\begin{aligned}
& \operatorname{trace}\left(\left(\operatorname{diag}\left(\mathbf{e}_{i}\right) \otimes \mathbf{I}_{\left(N_{t} \times N_{t}\right)}\right) \mathbf{W}\right) \leq \mathrm{P}_{i}^{o} \\
& \operatorname{rank}(\mathbf{W})=1 \\
& \mathbf{W} \succeq 0, \quad i=1, \ldots, M
\end{aligned}
$$

where $\mathbf{R}=\mathbf{H}^{H} \mathbf{H}, \mathbf{W}=\mathbf{w w}^{H}, \mathbf{R}_{\text {on }}=\left(\mathbf{H}_{\text {on }}^{o}\right)^{H} \mathbf{H}_{\text {on }}^{o}, \widehat{\mathbf{R}}_{\text {on }}=$ $\operatorname{diag}\left(\mathbf{e}_{n}\right) \otimes \mathbf{R}_{o n}$, and $\mathbf{e}_{n}=[0 \ldots 010 \ldots 0]^{T}$ is an $(M \times 1)$ unit vector with 1 as the $n^{\text {th }}$ element and zeros elsewhere. $\mathbf{I}_{\left(N_{t} \times N_{t}\right)}$ denotes the $\left(N_{t} \times N_{t}\right)$ identity matrix. By relaxing the rank 1 constraint, we obtain the semidefinite relaxation [20] of the problem:

$$
\begin{array}{cl}
\underset{\mathbf{w}_{1}^{o}, \ldots, \mathbf{w}_{M}^{o}}{\operatorname{minimize}} & \operatorname{trace}(\mathbf{R W}) \\
\text { subject to } & \operatorname{trace}\left(\left(\widehat{\mathbf{R}}_{o i}-\gamma_{i} \sum_{n \neq i} \widehat{\mathbf{R}}_{o n}\right) \mathbf{W}\right) \geq \gamma_{i} \sigma^{2} \\
& \operatorname{trace}\left(\left(\operatorname{diag}\left(\mathbf{e}_{i}\right) \otimes \mathbf{I}_{\left(N_{t} \times N_{t}\right)}\right) \mathbf{W}\right) \leq \mathrm{P}_{i}^{o} \\
& \mathbf{W} \succeq 0, \quad i=1, \ldots, M
\end{array}
$$

The SDP in (9) can be solved efficiently by software such as SeDuMi [14]. In case the resulting solution has a higher rank than one, we can use eigenvector approximation [13], in which the vector $\mathbf{w}$ is approximated as the eigenvector $\mathbf{q}_{1}$ corresponding to the largest eigenvalue of $\mathbf{W}$, scaled by the square root of the largest eigenvalue of $\mathbf{W}, \lambda_{1}$, i.e.,

$$
\begin{aligned}
\mathbf{W} & =\mathbf{w} \mathbf{w}^{H}=\sum_{i} \lambda_{i} \mathbf{q}_{i} \mathbf{q}_{i}^{H} \\
\mathbf{w} & \cong \sqrt{\lambda_{1}} \mathbf{q}_{1}
\end{aligned}
$$

After this step, the coefficients are determined using the conditions in (2)-(4) [12], as given by:

$$
\begin{aligned}
\alpha_{k m} & =\left(\mathbf{H}_{k m}^{o} \mathbf{w}_{m}^{o}\right)^{\dagger}\left(\mathbf{H}_{k 1}^{o} \mathbf{w}_{1}^{o}\right)^{H} \\
\left(\mathbf{H}_{k m}^{o} \mathbf{w}_{m}^{o}\right)^{\dagger} & =\left(\left(\mathbf{H}_{k m}^{o} \mathbf{w}_{m}^{o}\right)^{H}\left(\mathbf{H}_{k m}^{o} \mathbf{w}_{m}^{o}\right)\right)^{-1}\left(\mathbf{H}_{k m}^{o} \mathbf{w}_{m}^{o}\right)^{H}
\end{aligned}
$$

\section{Minimum Sum MSE With COORdinATED ZERO-FORCING}

Femtocell users can either cooperate and contribute interference alignment, which may increase the load on the backhaul or they can try to improve their own performance. As a suitable precoding-decoding scheme for the second case, each FBS may try to minimize the sum MSE of its own users, by zero-forcing the aligned macrocell users. A coordinated zeroforcing beamforming for SINR maximization was proposed in [15], which uses the ideas from [16] and [17].

We will use a precoding-decoding scheme that minimizes the sum MSE at the FBSs while zero-forcing the aligned interference from the macrocell users. The estimated bit of the $j^{\text {th }}$ user of the $k^{t h}$ femtocell is given as:

$$
\begin{aligned}
\hat{s}_{j}^{k}= & \sum_{i=1}^{U_{k}}\left(\mathbf{g}_{j}^{k}\right)^{H} \mathbf{H}_{k i}^{k} \mathbf{w}_{i}^{k} s_{i}^{k}+\sum_{\substack{f=1 \\
f \neq k}}^{F} \sum_{u=1}^{U_{f}}\left(\mathbf{g}_{j}^{k}\right)^{H} \mathbf{H}_{k u}^{f} \mathbf{w}_{u}^{f} s_{u}^{f} \\
& +\sum_{m=1}^{M}\left(\mathbf{g}_{j}^{k}\right)^{H} \mathbf{H}_{k m}^{o} \mathbf{w}_{m}^{o} s_{m}^{o}+\left(\mathbf{g}_{j}^{k}\right)^{H} \mathbf{n}_{k}
\end{aligned}
$$

where $\mathbf{g}_{j}^{k}$ is the decoding vector for the $j^{\text {th }}$ user of the $k^{t h}$ 
femtocell. Since the interference caused by other femtocells are very small compared to the intracell interference, for simplicity we will regard intercell femtocell interference as noise, which is given as:

$$
\tilde{\mathbf{n}}_{k}=\sum_{\substack{f=1 \\ f \neq k}}^{F} \sum_{u=1}^{U_{f}} \mathbf{H}_{k u}^{f} \mathbf{w}_{u}^{f} s_{u}^{f}+\mathbf{n}_{k}
$$

Using the conditions in (2)-(4) and (15), the minimum sum MSE at the FBS problem can be formulated as:

$$
\begin{array}{ll}
\underset{\substack{\mathbf{w}_{1}^{k}, \ldots, \mathbf{w}_{U_{k}}^{k} \\
\mathbf{g}_{1}^{k}, \ldots, \mathbf{g}_{U_{k}}^{k}}}{\operatorname{minimize}} & \sum_{j=1}^{U_{k}}\left\|\hat{s}_{j}^{k}-s_{j}^{k}\right\|^{2} \\
\text { s.t } & \left(\mathbf{g}_{j}^{k}\right)^{H} \mathbf{H}_{k 1}^{o} \mathbf{w}_{1}^{o}=0 \\
& \left(\mathbf{w}_{j}^{k}\right)^{H} \mathbf{w}_{j}^{k} \leq \mathrm{P}_{j}^{k} \quad j=1, \ldots, U_{k}
\end{array}
$$

or equivalently

$$
\begin{array}{ll}
\underset{\substack{\mathbf{w}_{1}^{k}, \ldots, \mathbf{w}_{U_{k}}^{k} \\
\mathbf{g}_{1}^{k}, \ldots, \mathbf{g}_{U_{k}}^{k}}}{\operatorname{minimize}} & \sum_{j=1}^{U_{k}}\left[\left|\left(\mathbf{g}_{j}^{k}\right)^{H} \mathbf{H}_{k j}^{k} \mathbf{w}_{j}^{k}-1\right|^{2}\right. \\
& \left.+\sum_{\substack{i=1 \\
i \neq j}}^{U_{k}}\left|\left(\mathbf{g}_{j}^{k}\right)^{H} \mathbf{H}_{k i}^{k} \mathbf{w}_{i}^{k}\right|^{2}+\left\|\mathbf{g}_{j}^{k}\right\|_{2}^{2} \tilde{\sigma}^{2}\right] \\
\text { s.t } & \left(\mathbf{g}_{j}^{k}\right)^{H} \mathbf{H}_{k 1}^{o} \mathbf{w}_{1}^{o}=0 \\
& \left(\mathbf{w}_{j}^{k}\right)^{H} \mathbf{w}_{j}^{k} \leq \mathrm{P}_{j}^{k} \quad j=1, \ldots, U_{k}
\end{array}
$$

where $\mathrm{P}_{j}^{k}$ is the maximum transmit power of the $j^{\text {th }}$ user of the $k^{\text {th }}$ femtocell, and $E\left\{\tilde{\mathbf{n}}_{k}\left(\tilde{\mathbf{n}}_{k}\right)^{H}\right\}=\tilde{\sigma}^{2} \mathbf{I}$. The zero forcing constraint in (18) implies that $\mathbf{g}_{j}^{k}$ should be in the null space of $\left(\mathbf{H}_{k 1}^{o} \mathbf{w}_{1}^{o}\right)$ [18], from which we can define a decoding vector such as:

$$
\mathbf{g}_{j}^{k}=\mathbf{U}_{k}^{o} \mathbf{v}_{j}^{k}
$$

where $\left[\mathbf{U}_{k}^{0} \mathbf{U}_{k}^{1}\right] \boldsymbol{\Lambda}_{k} \mathbf{V}_{k}$ is obtained from the SVD of $\mathbf{H}_{k 1}^{o} \mathbf{w}_{1}^{o}$ and the columns of $\mathbf{U}_{k}^{o}$ is a nullspace basis of $\mathbf{H}_{k 1}^{o} \mathbf{w}_{1}^{o}$. If we let $\left(\mathbf{U}_{k}^{0}\right)^{H} \mathbf{H}_{k j}^{k}=\widetilde{\mathbf{H}}_{k j}^{k}$, the problem in (18) is equal to:

$$
\begin{aligned}
& \underset{\substack{\mathbf{w}_{1}^{k}, \ldots, \mathbf{w}_{U_{k}}^{k} \\
\mathbf{v}_{1}^{k}, \ldots, \mathbf{v}_{U_{k}}^{k}}}{\operatorname{minimize}} \sum_{j=1}^{U_{k}}\left[\left|\left(\mathbf{v}_{j}^{k}\right)^{H} \widetilde{\mathbf{H}}_{k j}^{k} \mathbf{w}_{j}^{k}-1\right|^{2}\right. \\
& \left.+\sum_{\substack{i=1 \\
i \neq j}}^{U_{k}}\left|\left(\mathbf{v}_{j}^{k}\right)^{H} \widetilde{\mathbf{H}}_{k i}^{k} \mathbf{w}_{i}^{k}\right|^{2}+\left\|\mathbf{v}_{j}^{k}\right\|_{2}^{2} \tilde{\sigma}^{2}\right] \\
& \text { s.t } \quad\left(\mathbf{w}_{j}^{k}\right)^{H} \mathbf{w}_{j}^{k} \leq \mathrm{P}_{j}^{k} \quad j=1, \ldots, U_{k}
\end{aligned}
$$

The problem in (20) is convex in $\mathbf{w}_{j}^{k}$ if the all other $\mathbf{v}_{j}^{k}$ are fixed, and convex in $\mathbf{v}_{j}^{k}$ if all other $\mathbf{w}_{j}^{k}$ are fixed. Using this property, we can use an iterative algorithm by first fixing the decoding matrices and obtaining the precoding matrices, then fixing the precoding matrices to obtain the decoding matrices. An iterative procedure for obtaining the optimal coding vectors is used in [19] where the transmit precoding vector had unit norm. After writing the Lagrangian for the problem in (20), from the KKT conditions we have the optimal precoding and decoding vectors as:

$$
\begin{aligned}
& \mathbf{v}_{j}^{k}=\left(\sum_{i=1}^{U_{k}}\left(\widetilde{\mathbf{H}}_{k i}^{k} \mathbf{w}_{i}^{k}\right)\left(\widetilde{\mathbf{H}}_{k i}^{k} \mathbf{w}_{i}^{k}\right)^{H}+\tilde{\sigma}^{2} \mathbf{I}\right)^{-1} \widetilde{\mathbf{H}}_{k j}^{k} \mathbf{w}_{j}^{k} \\
& \mathbf{w}_{j}^{k}=\left(\sum_{i=1}^{U_{k}}\left(\widetilde{\mathbf{H}}_{k j}^{k}\right)^{H} \mathbf{v}_{i}^{k}\left(\mathbf{v}_{i}^{k}\right)^{H} \widetilde{\mathbf{H}}_{k j}^{k}+\mu_{j}^{k} \mathbf{I}\right)^{-1}\left(\widetilde{\mathbf{H}}_{k j}^{k}\right)^{H} \mathbf{v}_{j}^{k}
\end{aligned}
$$

for $j=1, \ldots, U_{k}$. We determine $\mu_{j}^{k}$ such that $\left(\mathbf{w}_{j}^{k}\right)^{H} \mathbf{w}_{j}^{k}=$ $\mathrm{P}_{j}^{k}$.

\section{Simulation Results}

Simulations are performed to compare the performance of the proposed macrocell interference alignment with that of the setting where macrocell users (MU) minimize their sum MSE at the MBS, without regard to femtocell users (FU). The MBS has a coverage radius of $2 \mathrm{~km}$, the group of FBSs close to each other is denoted by an area of $150 \mathrm{~m}$ radius, placed randomly according to a uniform distribution within the coverage radius of the MBS, and the MUs within this area apply interference alignment jointly. We consider 3 FBSs each with a radius of $30 \mathrm{~m}$ coverage. Each FBS has 3 users, and each mobile user has 4 transmit antennas. FBSs have 4 receive antennas. Noise power is assumed to be $-110 \mathrm{~dB}$. Power control at both FBS and MBS is used to compensate for the path loss. The maximum transmit power of each user is $1 \mathrm{~W}$.

The convergence of the proposed algorithm for 10 macrocell users and a minimum SINR requirement of 0.1 at the MBS is presented in Fig.3. The comparison of the SDP-IA scheme with the one with no interference alignment in terms of average BER versus the number of MUs interfering to the femtocell group is given in Fig.4. For the case when no interference alignment is applied, the only objective for the MUs is to minimize the sum MSE at the MBS. The number of MUs that can be aligned for different minimum SINR requirements at the MBS is depicted in Fig.5.

The results show that the performance of the FUs in terms of average BER is significantly better when compared to the case when the interfering MUs only consider their own performance and minimize the sum MSE at the MBS. It was observed in the simulations that, the received SINR constraints of the MUs in the second case do not satisfy a minimum and may cause an outage in voice applications. The feasibility of the minimum SINR constraints is a main limitation in this system: as the minimum SINR constraints of MUs are increased, the maximum number of MUs that can be aligned simultaneously decreases significantly.

\section{CONCLUSION}

In this paper, we considered interference management for a two-tier network with femtocells deployed within a macrocell. In particular, we opted for a design that mitigates interference caused by the macrocell users at multiple femtocell uplinks by using interference alignment (IA). Since, in this twotiered network, the interfering macrocell users need to have 


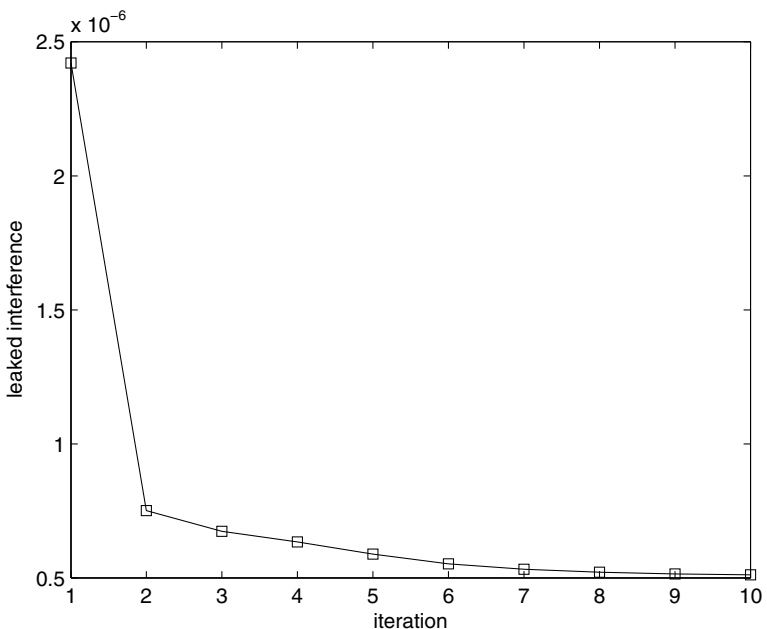

Fig. 3. Convergence results of the SDP-IA Algorithm

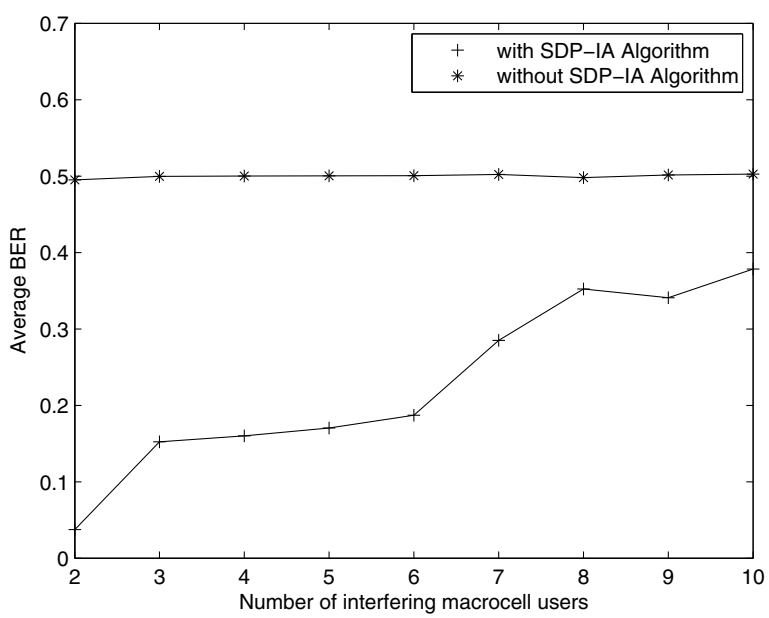

Fig. 4. Average BER of the femtocell users with and without SDP-IA Algorithm

their QoS requirements satisfied as well, we put forward an interference alignment algorithm with individual SINR constraints, in which we used the SDP approximation to a quadratically constraint quadratic problem (QCQP). The algorithm is applied to mitigate uplink macrocell interference in femtocell networks and numerical results are provided to demonstrate the advantage of the proposed design over the case when no interference alignment is done. Future work includes investigation of IA in tiered networks with further reduced complexity, and the impact of limited/noisy channel state information on the performance of IA.

\section{REFERENCES}

[1] V. Chandrasekhar, J. Andrews, A. Gatherer, "Femtocell networks: a survey," IEEE Commun. Mag., vol. 46, no. 9, pp. 59-67, Sept. 2008.

[2] Picochip, "The Case For Home Base Stations," White Paper, Apr. 2007.

[3] S. Verdu, "Multiuser Detection," Cambridge Univ. Press, 1998.

[4] R. Yates, "A framework for uplink power control in cellular radio systems," IEEE Journal on Sel. Areas Commun., vol. 13, no. 7, pp. 13411348, Sept. 1995.

[5] C. Rose, S. Ulukus, and R. Yates, "Wireless systems and interference avoidance," IEEE Trans. on Wireless Commun., vol. 1, no. 3, pp. 415428, July 2002.

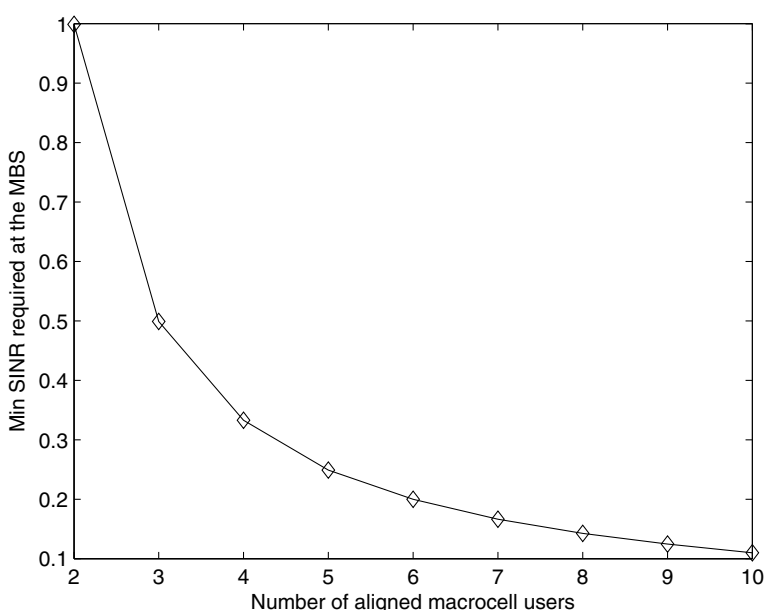

Fig. 5. Number of macrocell users that can be aligned subject to min SINR requirement at the MBS

[6] W. M. Jang, B. R. Vojcic, and R. L. Pickholtz, ”Joint transmitter-receiver optimization in synchronous multiuser communications over multipath channels," IEEE Trans. on Commun., vol. 46, pp. 269278, Feb. 1998.

[7] V. R. Cadambe and S. A. Jafar, "Interference alignment and degrees of freedom region for the $\mathrm{K}$ user interference channel," IEEE Trans. on Inf. Theory, vol. 54, no. 8, Aug. 2008.

[8] K. S. Gomadam, V. R. Cadambe, and S. A. Jafar, "Approaching the capacity of wireless networks through distributed interference alignment," arXiv, no. 0803.3816, 2008.

[9] D.A. Schmidt, C. Shi, R.A. Berry, M.L. Honig, W. Utschick, "Minimum Mean Squared Error interference alignment," 2009 Conference Record of the Forty-Third Asilomar Conference on Signals, Systems and Computers, pp. 1106-1110, 1-4 Nov. 2009.

[10] S. W. Peters and R. W Heath, Jr., "Interference Alignment via Alternating Minimization," Proc. of IEEE Int. Conf. on Acoustics, Speech, and Signal Processing, pp. 2445 2448, Apr. 2009.

[11] ITU-R Recommendation M.1225: "Guidelines for evaluation of radio transmission technologies for IMT-2000," Feb. 1997.

[12] H. Yu, Y. Sung, "Least Squares Approach to Joint Beam Design for Interference Alignment in Multiuser Multi-Input Multi-Output Interference Channels," IEEE Trans. on Signal Proc., vol. 58, no. 9, Sept. 2010.

[13] Z. Luo, W. Ma, A.M. So, Y. Ye; S. Zhang, "Semidefinite Relaxation of Quadratic Optimization Problems," IEEE Sig. Proc. Mag., vol. 27, no. 3, pp. 20-34, May 2010.

[14] D. Peaucelle, D. Henrion, Y. Labit, and K. Taitz, "Users Guide for SeDuMi Interface 1.04," LAAS-CNRS, Toulouse, 2002.

[15] X. Sun, L. J. Cimini, L. J. Greenstein, D. S. Chant, and J. Kruys, "Coordinated zero-forcing beamforming in multipoint MIMO networks for backhaul applications," IEEE Military Commun. Conf., pp. 1-7, 1821 Oct. 2009.

[16] S. Ulukus and R. D. Yates, "Iterative Construction of Optimum Signature Sequence Sets in Synchronous CDMA Systems," IEEE Trans. on Inform. Theory, vol. 47, no. 5, pp. 1989-1998, July 2001.

[17] Z. Pan, Kai-Kit Wong, and Tung-Sang Ng, "Generalized multiuser orthogonal space-division multiplexing," IEEE Trans. on Wireless Commun., vol. 3, no. 6, pp. 1969-1973, Nov. 2004.

[18] Q. H. Spencer, A. L. Swindlehurst, and M. Haardt, "Zero-forcing methods for downlink spatial multiplexing in multiuser MIMO channels," IEEE Trans. on Signal Proc., vol. 52, no. 2, pp. 461-471, Feb. 2004.

[19] S. Serbetli, A. Yener, "Transceiver optimization for multiuser MIMO systems," IEEE Trans. on Signal Proc., vol. 52, no. 1, pp. 214-226, Jan. 2004.

[20] S. Boyd and L. Vandenberghe, "Convex Optimization," Cambridge Univ. Press, 2004.

[21] M. Kojima, L. Tuncel, "On the Finite Convergence of Successive SDP Relaxation Methods," European Journal of Operations Research, vol. 143, no. 2 pp. 325341, Dec. 2002. 